\title{
Efeito da idade do touro e do período de colheita de sêmen sobre as características físicas e morfológicas do sêmen de bovinos de raças européias e zebuínas
}

\author{
Antônio Rodrigues da Silva ${ }^{1}$, Antonio Sergio Ferraudo², Dilermando Perecin², \\ Vera Fernanda Martins Hossepian de Lima $^{3}$
}

\author{
${ }^{1}$ Departamento de Zootecnia da UFMT, CEP: 78735-901, Rondonópolis, MT, Brasil. \\ 2 Departamento de Ciências Exatas da FCAV, Unesp, CEP: 14884-900, Jaboticabal, SP, Brasil. \\ ${ }^{3}$ Departamento de Medicina Veterinária Preventiva e Reprodução Animal da FCAV, Unesp, CEP: 14884-900, Jaboticabal, SP, Brasil.
}

RESUMO - Este trabalho foi realizado com o objetivo de avaliar o efeito da idade de touros europeus e zebuínos e do período de colheita do sêmen sobre as características físicas e morfológicas do sêmen desses animais produzido em uma central de inseminação no período de 1993 a 1999. Os dados de produção de sêmen dos touros foram agrupados em cinco classes de idade (12 a 36 meses, 37 a 60 meses, 61 a 84 meses, 85 a 108 meses e 109 a 142 meses) e quatro períodos de colheita (período 1: dezembro a fevereiro; período 2: março a maio; período 3: junho a agosto e período 4: setembro a novembro). As classes de idade determinaram diferenças significativas no volume, turbilhonamento espermático, nas anormalidades primárias, secundárias, terciárias e totais, na integridade de acrossoma e na quantidade média de doses por ejaculado, cujos valores foram maiores nos zebuínos no período 4 (setembro a outubro). As maiores porcentagens de anormalidades totais, nas duas subespécies, foram observadas nos animais mais jovens (12 a 36 meses) e nos mais velhos (109 e 142 meses). Os zebuínos mais velhos produziram sêmen mais concentrado e maiores quantidades médias de doses por ejaculado. Em touros europeus, o sêmen menos concentrado e as maiores porcentagens de anormalidades espermáticas foram observadas no período 1 (dezembro a fevereiro), consequentemente, menores quantidades de doses de sêmen por ejaculado foram produzidas por essa subespécie, o que pode ter sido um efeito do estresse calórico sofrido por estes animais antes da colheita de sêmen. A idade e o período de colheita influenciam na qualidade do sêmen de touros doadores mantidos em regime de colheita para comercialização.

Palavras-chave: bovino, qualidade do sêmen, sazonalidade

\section{Effect of the age of the bull and the semen collection periods on physical and morphological semen characteristics of European and zebu breeds}

\footnotetext{
ABSTRACT - The objective of this study was to evaluate the effect of the European and Zebu age of the bull and semen collection periods on the physical and morphological characteristics of the semen produced by animals in an artificial insemination station, from 1993 to 1999. The semen production data were grouped in five age classes: 12 to 36 months, 37 to 60 months, 61 to 84 months, 85 to 108 months and 109 to 142 months; and in four collection periods: 1 - December to February; 2 - March to May, 3 - June to August and 4 - September to November. Significant effects of age and collection periods on semen characteristics were observed. Volume, sperm turbulence, primary, secondary, tertiary and total abnormalities, acrosome integrity and the average amount of doses per ejaculate showed statistical differences among age classes, with higher values for Zebu in period 4 (September to October). For both subspecies, the highest percentage of total abnormalities was observed in the younger (12 to 36 month-old) and older (109 to 142 month-old) groups of animals. The older Zebu group produced more concentrated semen and showed high average amount of doses per ejaculate. The effect of age on semen characteristics could be observed during the productive and reproductive life of the cattle studied, except for sperm motility and sperm vigor of fresh semen. Less concentrated semen and high percentage of abnormal spermatozoa were observed in the European bulls during season 1 (December to February), as a consequence, a small quantity of semen doses per ejaculate was produced by this subspecies. It could be an effect of the heat stress suffered by these animal immediately before semen collection.
}

Key Words: cattle semen, seasonality, semen quality 


\section{Introdução}

A maior parte dos touros doadores de sêmen é selecionada pelo pedigree, pelas características de conformação e desempenho ponderal individual e pelo resultado de exames andrológicos. Nesses touros, pode ser observado o efeito das estações do ano sobre as características seminais (Fonseca et al., 1992). A permanência desses animais em empresas de colheita e congelamento de sêmen não garante que o efeito da estação do ano seja reduzido e que os touros produzam ejaculados com sêmen de boa qualidade quando submetidos a regime permanente de colheita de sêmen (Rekwot et al., 1987; Sekoni et al., 1988; Stålhammar et al., 1989; Söderquist et al., 1996).

Os ejaculados produzidos no início da vida reprodutiva dos bovinos apresentam em média 50 milhões de espermatozóides por mililitro e motilidade espermática de $10 \%$. Esses eventos começam a partir dos 8 meses de idade em bovinos de raças taurinas e zebuínas e, normalmente, os primeiros ejaculados têm grande porcentagem de anormalidades espermáticas. Entretanto, com o pasar da idade, diminui a porcentagem de anormalidades primárias (anomalias no acrossoma, cabeça pequena e piriforme), como resultado de alterações na espermatogênese (Johnson, 1997).

Söderquist et al. (1996) e Dairra et al. (1997) avaliaram a produção seminal de touros da subespécie Bos taurus taurus, de 12 a 60 meses de idade, mantidos em central de inseminação artificial divididos em classes de idade, e observaram efeito significativo da idade $(\mathrm{P}<0,05)$ sobre $\mathrm{o}$ volume, a concentração, a motilidade e as anormalidades espermáticas. Entretanto, de acordo com esses autores, as anormalidades primárias e secundárias (cabeças anormais dos espermatozóides e caudas enroladas) e os defeitos estruturais (defeitos na peça intermediária, cabeça decapitada e gota citoplasmática) foram mais frequentes.

Outros fatores podem afetar a qualidade do sêmen bovino produzido ao longo do ano. Segundo Mathevon et al. (1998), altas temperaturas nos períodos anteriores à colheita afetaram significativamente todas as características do sêmen de bovinos jovens e não influenciaram o volume e a motilidade espermática nos animais mais velhos da subespécie Bos taurus taurus. Conforme relatado por Silva (2000), os efeitos nocivos do estresse térmico sobre o sêmen de touros ocorrem nas três primeiras semanas da espermatogênese e ocorrem inclusive em animais adaptados a climas tropicais.

Nos últimos 30 anos, centrais de inseminação artificial têm trabalhado para produzir maiores quantidades de sêmen de touros oriundos de rebanhos selecionados para aumento do número de animais nascidos a partir de inseminação artificial, o que possibilitaria melhores resultados em um mercado tão competitivo. Este estudo foi realizado com o objetivo de avaliar o efeito da idade do touro e do período de colheita de sêmen nas características físicas e morfológicas de sêmen produzido por touros da central de inseminação artificial.

\section{Material e Métodos}

Foram utilizados registros de produção de sêmen de 42 touros (22 Bos taurus taurus, Europeus, e 20 Bos taurus indicus, Zebu). Os dados foram coletados nos períodos em que cada touro permanecia em regime de colheita de sêmen na Central de Inseminação Artificial. As colheitas foram feitas pelo método de vagina artificial duas vezes por semana para análise das seguintes características: volume de sêmen ejaculado; turbilhonamento espermático; e concentração, motilidade e vigor espermático do sêmen fresco; motilidade espermática e vigor imediatamente após descongelamento e motilidade e vigor espermático 3 horas após descongelamento. As anormalidades espermáticas primárias, secundárias, terciárias e totais e a integridade do acrossoma 3 horas após descongelamento foram estimadas em porcentagem. A quantidade média de doses por ejaculado foi obtida segundo protocolo adotado pela central de inseminação artificial.

Os dados referentes ao sêmen foram divididos em cinco classes de idade (12 a 36 meses 37 a 60 meses, 61 a 84 meses, 85 a 108 meses e 109 a 142 meses) e quatro períodos de colheita do sêmen, ou estações (período 1: dezembro a fevereiro; período 2: março a maio; período 3: junho a agosto e período 4: setembro a novembro).

Para identificar o efeito das classes de idade e dos períodos de colheita em cada característica do sêmen, foi utilizado o seguinte modelo estatístico:

$$
\begin{gathered}
\mathrm{Y}_{\mathrm{ijk}}=\mu+\operatorname{Sesp}_{\mathrm{i}}+\text { Est }_{\mathrm{k}}+\text { Claid }_{\mathrm{j}}+\left(\operatorname{Sesp}_{\mathrm{i}} \times \text { Est }_{\mathrm{k}}\right)+\left(\operatorname{Sesp}_{\mathrm{i}}\right. \\
\left.\times \text { Claid }_{\mathrm{J}}\right)+\mathrm{e}_{\mathrm{ijk}},
\end{gathered}
$$

em que: $Y_{\mathrm{ijk}}=$ característica física ou morfológica do sêmen da i-ésima subespécie ( $\mathrm{i}=1,2)$, na j-ésima classe de idade ( $\mathrm{j}=1,2,3,4,5)$ no $\mathrm{k}$-ésimo período $(\mathrm{k}=1,2,3,4) ; \mu$ = média geral; $\operatorname{Sesp}_{\mathrm{i}}=$ efeito da i-ésima subespécie (bovino europeu ou zebu); Est $_{\mathrm{k}}=$ efeito da k-ésima estação de colheita de sêmen; Claid $_{j}=$ efeito da j-ésima classe de idade (meses); $\operatorname{Sesp}_{\mathrm{i}} \times$ Est $_{\mathrm{k}}=$ interação do efeito da i-ésima subespécie com a k-ésima estação de colheita; $\operatorname{Sesp}_{i} \times$ Claid $_{j}=$ interação efeito da i-ésima subespécie $\times$ j-ésima classe de idade; $\mathrm{e}_{\mathrm{ijkm}}=$ erro aleatório assumido $\operatorname{NID}\left(0, \sigma^{2}\right)$. 
As análises estatísticas univariadas foram processadas no Statistical Analysis System - SAS (1996), versão 6.12, utilizando-se o procedimento Proc GLM e as médias foram comparadas pelo teste Tukey $(\mathrm{P}<0,05)$.

\section{Resultados e Discussão}

A interação subespécie $\times$ classe de idade teve efeito significativo sobre o volume do ejaculado $(\mathrm{P}<0,05)$, o turbilhonamento espermático $(\mathrm{P}<0,01)$ e a concentração espermática $(\mathrm{P}<0,01)$, assim, o efeito de subespécie sobre essas características foi analisado dentro de cada classe de idade. Os resultados observados neste estudo confirmam os encontrados por Stålhammar et al. (1989) e Dairra et al. (1997), que investigaram a influência da idade de bovinos europeus sobre a qualidade do sêmen e constataram que a idade do touro afetou significativamente o volume de sêmen ejaculado, a concentração espermática, as anormalidades espermáticas e a quantidade de doses por ejaculado.

$O$ volume de sêmen nos touros europeus não aumentou significativamente com a idade, enquanto, nos touros zebuínos, o aumento foi significativo nos animais da classe 5. Esses resultados corroboram os demonstrados por Brito et al. (2002a), que não encontraram diferença no volume de sêmen entre essas subespécies. Brito et al. (2002b) e Fuerst-Waltl et al. (2006) observaram aumento de volume com a idade em touros europeus em regime de colheita em central.

No sêmen da subespécie Bos taurus indicus, a maior média de turbilhonamento $(4,32)$ foi observada na classe 5 (Tabela 1), o que pode indicar maior qualidade física do sêmen, segundo as normas sugeridas pelo Ministério da Agricultura, Pecuária e Abastecimento e citadas pelo Colégio Brasileiro de Reprodução Animal (1998) (valores de turbilhonamento espermático acima de 3 pontos).

A concentração espermática não aumentou significativamente com a idade nos touros europeus. Nos touros zebuínos, esta característica decresceu da classe de idade 1 até a 3 e, a partir da classe 3, aumentou significativamente. A concentração espermática no sêmen desses animais foi maior que nos dos touros europeus em todas as classes de idade e foi maior no sêmen dos animais mais jovens e dos mais velhos. Assim, as quantidades médias de doses por ejaculado foram maiores nos touros zebuínos (Tabela 1) e essas variações na concentração espermática podem estar relacionadas ao efeito da subespécie e ao menor volume do ejaculado das raças zebuínas. O menor volume do ejaculado das raças zebuínas pode ter ocasionado aumento da concentração em comparação a touros europeus, como demonstrado por Brito et al. (2002a,b).

A quantidade média de doses por ejaculado diferiu $(\mathrm{P}<0,05)$ entre as classes de idade (Tabela 1$)$. Nos touros europeus, a quantidade de doses aumentou da classe de idade 1 até 4 , decrescendo da classe 4 para a 5 (Tabela 1), enquanto, nos touros zebuínos, a quantidade de doses aumentou da classe de idade 1 para a 2, decrescendo na idade 3 e aumentando novamente nas demais classes.

O vigor espermático no sêmen fresco e imediatamente após descongelamento não diferiu significativamente, entretanto, quando avaliado 3 horas após descongelamento, apresentou diferenças $(\mathrm{P}<0,01)$ entre as subespécies, as estações, as classes de idade e foi influenciado pela interação subespécie $\times$ classe de idade.

Os valores encontrados para anormalidades espermáticas primárias diferiram significativamente entre subespécies e estações e foram influenciados pela interação subespécie $\times$ classe de idade $(\mathrm{P}<0,01)$. A porcentagem de anormalidades secundárias (gotas citoplasmáticas e caudas enroladas) foi influenciada $(\mathrm{P}<0,01)$ pelas subespécies e classes de idade e a de anormalidades terciárias, pelas subespécies e estações. A porcentagem de anormalidades totais diferiu significativamente entre classes de idade, estação e subespécie. Os resultados observados na classe de idade 1 (12 a 36 meses) para anormalidades totais foram semelhantes aos encontrados por Söderquist et al. (1996),

Tabela 1 - Características do sêmen de bovinos europeus e zebuínos de cada classe de idade

\begin{tabular}{|c|c|c|c|c|c|c|c|c|}
\hline \multirow[t]{2}{*}{$\begin{array}{l}\text { Classe de } \\
\text { idade (meses) }\end{array}$} & \multicolumn{2}{|c|}{ Volume(mL) } & \multicolumn{2}{|c|}{ Turbilhonamento } & \multicolumn{2}{|c|}{$\begin{array}{c}\text { Concentração espermática } \\
\times 10^{9} / \mathrm{mL}\end{array}$} & \multicolumn{2}{|c|}{$\begin{array}{l}\text { Quantidade média de } \\
\text { doses/ejaculado }\end{array}$} \\
\hline & Europeu & Zebu & Europeu & Zebu & Europeu & Zebu & Europeu & Zebu \\
\hline 37 a 60 & 5,93Ab & $6,52 \mathrm{Aa}$ & $3,52 \mathrm{Aa}$ & $3,60 \mathrm{Aa}$ & $1,400 \mathrm{Aa}$ & $1,402 \mathrm{Ab}$ & $145,9 \mathrm{Ab}$ & $162,4 \mathrm{Ab}$ \\
\hline 61 a 84 & $6,64 \mathrm{Aa}$ & $6,35 \mathrm{Aa}$ & $3,37 \mathrm{Aa}$ & $3,47 \mathrm{Aa}$ & $1,367 \mathrm{Aa}$ & $1,355 \mathrm{Ab}$ & $164,6 \mathrm{Aab}$ & $157,2 \mathrm{Ab}$ \\
\hline 85 a 108 & $6,71 \mathrm{Aa}$ & $6,31 \mathrm{Aa}$ & $3,26 \mathrm{Aa}$ & $3,81 \mathrm{Ba}$ & $1,295 \mathrm{Aa}$ & $1,554 \mathrm{Ba}$ & $172,8 \mathrm{Aa}$ & $175,8 \mathrm{Aa}$ \\
\hline
\end{tabular}

Médias seguidas por letras maiúsculas diferentes nas colunas diferem entre si e seguidas por letra minúsculas diferentes nas linhas diferem $(\mathrm{P}<0,05)$ entre si pelo teste Tukey. 
que avaliaram a produção de sêmen de touros da subespécie Bos taurus taurus mantidos em regime de colheita em central de inseminação artificial na Suécia e encontraram diferenças $(\mathrm{P}<0,01)$ para idade nesta característica. Estão de acordo também com os citados por Johnson (1997), que relatou a avaliação de sêmen de bovinos em três grupos de idade ( 2 a 3, 3 a 4 e 4 a 5 anos de idade) e constatou que os espermatozóides dos touros de 3 a 4 anos de idade tiveram menos anormalidades totais em comparação aos dos touros de outros grupos.

As porcentagens de anormalidades primárias nos touros de 12 a 36 meses de idade foram maiores para bovinos europeus (7,33\%) e, na classe 5, para zebuínos (5,91\%) (Tabela 2). A porcentagem de integridade de acrossoma diferiu $(\mathrm{P}<0,05)$ apenas entre os touros mais velhos das duas subespécies. Esta característica pode permitir estimar quantos espermatozóides podem estar vivos e intactos 3 horas após o descongelamento do sêmen.

Não foram observadas diferenças significativas na interação subespécie × estação (período de colheita). Das características estudadas, foram significativas a motilidade espermática imediatamente após descongelamento $(\mathrm{P}<0,01)$, a quantidade média de doses por ejaculado $(\mathrm{P}<0,05)$ e o vigor espermático 3 horas após descongelamento $(\mathrm{P}<0,01)$. Os resultados de motilidade e vigor estão de acordo com as recomendações do Ministério da Agricultura, citados pelo Colégio Brasileiro de Reprodução Animal (1998), para sêmen de touros em centrais de inseminação artificial.
As quantidades médias de doses por ejaculado observadas na subespécie Bos taurus taurus nos períodos de colheita foram de 147,1; 148,1; 163,9 e 172,9 doses e, na subespécie Bos taurus indicus, foram 162,1; 145,2; 157,0 e 179,9 doses por ejaculado, respectivamente (Tabela 1). Maiores quantidades de doses foram encontradas no período de setembro a novembro nas duas subespécies. Esses resultados estão de acordo com os encontrados por Sekoni et al. (1988) para touros zebuínos na Nigéria durante o inverno (julho a setembro). Entretanto, não confirmam os estudos realizados por Stålhammar et al. (1989), na Suécia, com bovinos europeus, nos quais foram encontradas maiores quantidades de doses por ejaculado no verão (junho a agosto).

As anormalidades primárias, terciárias e totais foram estatisticamente significativas $(\mathrm{P}<0,01)$ nos respectivos períodos de colheita estudados (Tabela 3 ). As quantidades médias de doses por ejaculado observadas na subespécie Bos taurus taurus nos períodos de colheita foram de 147,1; 148,1; 163,9 e 172,9 doses (Tabela 3). Os bovinos da subespécie Bos taurus indicus nos períodos de dezembro a janeiro, de março a maio, de junho a agosto e de setembro a novembro produziram 162,1; 145,2; 157,0 e 179,9 doses, respectivamente. Maiores quantidades de doses foram encontradas no período de setembro a outubro nas duas subespécies, provavelmente em razão do início do período das águas, que favorece o crescimento do pasto e melhora o aporte forrageiro aos animais.

Tabela 2 - Características do sêmen de bovinos europeus e zebuínos de diversas classes de idade

\begin{tabular}{|c|c|c|c|c|c|c|}
\hline Classe de idade & \multicolumn{2}{|c|}{ Vigor 3 horas após descongelamento (\%) } & \multicolumn{2}{|c|}{ Anormalidades primárias } & \multicolumn{2}{|c|}{ Integridade deacrossoma, \% } \\
\hline 12 а 36 & $3,14 \mathrm{Ab}$ & $3,57 \mathrm{Ba}$ & 7,33Аa & $4,28 \mathrm{Bb}$ & 49,83Аа & $50,18 \mathrm{Aa}$ \\
\hline 61 a 84 & $3,39 \mathrm{Aa}$ & $3,41 \mathrm{Aa}$ & $6,06 \mathrm{Ab}$ & $5,28 \mathrm{Ba}$ & $49,59 \mathrm{Aa}$ & $49,40 \mathrm{Aa}$ \\
\hline 85 a 108 & $3,15 \mathrm{Ab}$ & 3,46Ba & $5,98 \mathrm{Ab}$ & $5,27 \mathrm{Ba}$ & $48,72 \mathrm{Ab}$ & $49,47 \mathrm{Ba}$ \\
\hline 109 а 142 & $3,25 \mathrm{Ab}$ & $3,10 \mathrm{Ab}$ & 4,21Aa & 5,91Ba & $50,00 \mathrm{Aa}$ & $47,96 \mathrm{Bb}$ \\
\hline
\end{tabular}

Médias seguidas de letras maiúsculas diferentes nas colunas diferem entre si e seguidas de letra minúscula diferentes nas linhas diferem $(\mathrm{P}<0,05)$ entre si pelo teste Tukey.

Tabela 3 - Características de anormalidades espermáticas no sêmen de bovinos europeus e zebuínos

\begin{tabular}{|c|c|c|c|c|c|c|c|c|c|c|}
\hline \multirow[t]{2}{*}{ Período decolheita } & \multicolumn{2}{|c|}{$\begin{array}{c}\text { Anormalidades } \\
\text { primárias }\end{array}$} & \multicolumn{2}{|c|}{$\begin{array}{l}\text { Anormalidades } \\
\text { secundárias }\end{array}$} & \multicolumn{2}{|c|}{$\begin{array}{c}\text { Anormalidades } \\
\text { terciárias }\end{array}$} & \multicolumn{2}{|c|}{$\begin{array}{c}\text { Anormalidades } \\
\text { totais }\end{array}$} & \multicolumn{2}{|c|}{$\begin{array}{c}\text { Quantidade média de } \\
\text { doses/ejaculado }\end{array}$} \\
\hline & Europeu & Zebu & Europeu & Zebu & Europeu & Zebu & Europeu & Zebu & Europeu & Zebu \\
\hline Março a maio & $6,00 \mathrm{Aa}$ & $5,34 \mathrm{Aa}$ & 4,33Aa & $4,83 \mathrm{Aa}$ & $2,11 \mathrm{Aa}$ & $1,45 \mathrm{Aa}$ & $12,44 \mathrm{Aa}$ & $11,61 \mathrm{Aa}$ & 148,1 & 145,2 \\
\hline Junho a agosto & 5,99Аа & $4,32 \mathrm{Ba}$ & 3,91Aa & $4,93 \mathrm{Aa}$ & $1,78 \mathrm{Ab}$ & $1,46 \mathrm{Aa}$ & $11,69 \mathrm{Aa}$ & $10,71 \mathrm{Aa}$ & 163,9 & 157,0 \\
\hline
\end{tabular}

novembro

Médias seguidas por letras maiúsculas diferentes nas colunas diferem entre si e seguidas por letra minúsculas diferentes nas linhas diferem (P<0,05) entre si pelo teste Tukey. 
Os resultados para anormalidades totais foram superiores aos encontrados por Söderquist et al. (1996), que verificaram diferenças significativas $(\mathrm{P}<0,05)$ nos períodos de colheita de sêmen para defeitos totais em bovinos europeus. Em bovinos zebuínos, no entanto, essas anormalidades foram menores que as encontradas no período de janeiro a abril por Sekoni et al. (1988), que observaram diferenças significativas nas características seminais de zebuínos na Nigéria nos períodos de janeiro a abril, maio a agosto e de setembro a dezembro.

\section{Conclusões}

A idade e o período de colheita de sêmen podem ser utilizados para estimar a qualidade do sêmen (características físicas e morfológicas) e a quantidade de doses produzidas por touros doadores de sêmen em centrais de inseminação artificial. O volume, o turbilhonamento, a concentração espermática, a quantidade média de doses por ejaculado, o vigor espermático 3 horas após o descongelamento e a porcentagem de integridade de acrossoma foram influenciados pela idade do touro nas raças zebuínas. Entretanto, das características morfológicas estudadas, apenas as anormalidades primárias foram afetadas pelas subespécies em algumas classes de idade.

\section{Literatura Citada}

BRITO, L.F.; SILVA, A.E.; RODRIGUES, L.H. et al. Effects of enviromental factors, age and genotype on sperm production and sêmen quality in Bos indicus and Bos taurus AI bulls in Brazil. Animal Reproduction Science, v.70, p.181-90, 2002a.

BRITO, L.F.; SILVA, A.E.; RODRIGUES, L.H. et al. Effect of age and genetic group on characteristics of the scrotum, testes and testicular vascular cones, and on sperm production and semen in AI bulls in Brazil. Theriogenology, v.58, p.1175-86, 2002b. COLÉGIO BRASILEIRO DE REPRODUÇÃO ANIMAL - CBRA. Manual para exame andrológico e avaliação de sêmen animal. Belo Horizonte: 1998. p.49.

DAIRRA, M.S.; PARE, J.P.; ROY, G. Genetic and environmental factores affecting semen quality of young Holstein bulls. Canadian Journal Animal Science, v.77, n.1, p.77-85, 1997. FONSECA, V.O.; CRUDELI, G.A.; COSTA E SILVA, E.V. et al. Aptidão reprodutiva de touros da raça Nelore. Efeito de diferentes estações do ano sobre as características seminais, circunferências escrotal e fertilidade. Arquivo Brasileiro de Medicina Veterinária e Zootecnia, v.44, n.1, p.7-15, 1992.

FUERST-WALTL, B.; SCHWARZENBACHER, H., PERNER, C. et al. Effects of age and enviromental factors on semen production and semen quality of Austrian Simental bulls. Animal Reproduction Science, v.95, p.27-37, 2006.

JOHNSON, W.H. The significance to bull fertility of morphologically abnormal sperm. Veterinary Clinical North American: Food Animal Practical, v.13, n.2, p.255-270, 1997.

MATHEVON, M.; BUHR, M.M.; DEKKERS, J.C. Environmental, management, and genetic factors affecting semen production in Holstein bulls. Journal Dairy Science, v.81, n.12, p.3321-3330, 1998.

REKWOT, P.I.; VOH JR., A.A.; OYEPIDE, D.E. et al. Influence of season on characteristics of the ejaculate from bulls in Artificial Insemination Center in Nigeria. Animal Reproduction Science, v.14, p.187-94, 1987.

STATISTICAL ANALYSIS SYSTEM - SAS. SAS/STAT user's guide: Stat, version 6.11. Cary, 1996. 1 CD-ROM.

SEKONI, V.O.; GUSTAFSON, B.K. The incidence of sperm abnormalities in dairy bulls and effects of age and season. In: INTERNATIONAL CONGRESS ANIMAL REPRODUTION AND ARTIFICIAL INSEMINATION, 9., 1988, Shika. Anais.. Shika: International Society of Animal Reproduction, 1988. v.3. p.284.

SILVA, R.G. Introdução à bioclimatologia animal. São Paulo: Nobel, 2000. p.16-26.

SÖDERQUIST, L.; JANSON, L.; HAARD, M. et al. Influence of season, age, breed and some other factores on the variation in sperm morphological abnormalities in Swedish dairy A.I. bulls. Animal Reprodution Science, v.44, p.91-8, 1996.

STÅLHAMMAR, E-M.; JANSON, L.; PHILIPSSON, J. Genetics studies on fertility in A.I. bulls. I. Age, season and genetic effects on semen characteristics in young bulls. Animal Reprodution Science, v.19, p.1-17, 1989. 